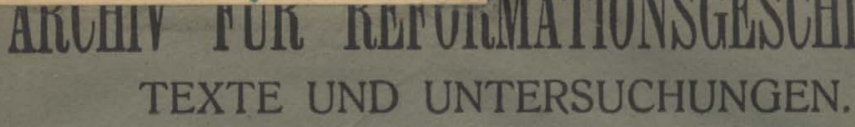

Im Auftrag des Vereins für Reformationsgeschichte herausgegeben von

Walter Friedensburg und Otto Scheel.

Nr. $105 / 106$. XXVII. Jahrgang. Heft $1 / 2$.

\section{Sonderabdruck}

\section{Ih. Wotschle}

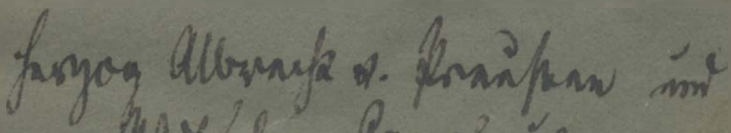

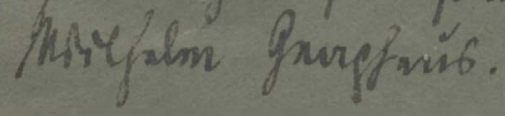

Leipzig 1930

Verlag von $M$. Heinsius Nachfolger

Eger \& Sievers. 
Rückseite: Anna Guldemunds zu Weimar bittet ieerlich and aff Leben lang umb $20 \mathrm{fl}$. und 1 malder korn. Es wirtt nichtts yn der rentterey von dyser suplycantten suchung befunden, dan es wissen sich Guntter Herwagen und her Johann Kestner zw erynnern, das di fraw XXX gulden ausm Closter Heustorff vom probst empfangen, es hatt aber der probst kein rechnung gehalden ader gethann, daramb nigs davon ihn der Rentterey $z w$ befinden. Jhedoch statt es $\mathrm{zw}$ meyns gnedigsten herren gnedigsten gefallen, was der frauen auff Ihr pidtt gereicht werden sahl. Rentmaister scr. Kurfürsten Johann Friedrichs Antwort aber vom 5. Januar 1544 aus Weimar war eine Anweisung an seinen thitringischen Rentmeister, die Guldemund mit den ausgebetenen 20 fl. zufriedenstellen za wollen. ${ }^{1}$ )

Ernestinisches Ges. Archiv Weimar, Reg. O0. pag. $792 \mathrm{Nr} .454$.

\title{
Herzog Albrecht von Preußen und Wilhelm Gnapheus.
}

\author{
Ein Nachtrag. \\ Von Theodor Wotschke.
}

Reusch in dem Elbinger Gymnasialprogramm 1877 und Tschackert in seinem Urkundeubuche zur Reformationsgeschichte des Herzogtums Preußen haben die Elbinger und Königsberger Tätigkeit des niederländischen Humanisten Wilhelm Gnapheus in das helle Licht der Geschichte geritckt, uber sein Leben and Wirken eingehend berichtet. So viel Neues aber besonders Tschackert bringt, das Königsberger Archiv bat er nicht völlig ausgeschöpft, deshalb vermag ich einen kleinen Nachtrag zu seinen Forschungen zu bringen ${ }^{2}$ ).

Im Jahre 1531 war Gnapheus, der ob seiner Hinneigung zum Evangelium in den Niederlanden mehr als einmal Gefängnisstrafe erlitten batte, nach Elbing gekommen and hier im Winter 1535/36 Rektor des unlängst gegründeten Gymnasiums geworden. Auf seine Empfehlung suchte der Rat der Stadt den Hieronymus Phristinus Nestenus, der in Königsberg an der Schule diente, sich aber ehemals Elbing verpflichtet hatte, als zweiten Lehrer zu gewinnen. Er schrieb an ihn, und Nesthenus war geneigt, dem Rufe zu folgen. Er

1) Auf wiederholtes Ansuchen als Witwe (26. Nov. 1552 n. 4. März 1553) wird Anna Guldemund ron Johann Friedrich d. Gr. am 4. März u. 28. Dezbr. 1553 and ron Johann Friedrich d. M. und Johann Wilhelm am 18. April 1558 mit Zulagen aus Kl. Oberweimar bedacht. Ernest. Ges. A. Weimar, KK. 650.

2) Sämtliche Nachrichten sind dem Staatsarchive in Königsberg entnommen. 
wandte sich an den Herzog und bat um seine Entlassung. Aber er erhielt sie nicht. Der Herzog wollte den tiuchtigen Lehrer nicht missen. Am 25. Mai 1536 schrieb er deshalb an den Elbinger Rat $^{1}$ ). Er blieb bei seiner ablehneuden Haltung, auch als der Rat nun schon unter dem 29. sich direkt an ihn wandte und um Entlassung des Nesthenus bat. Er wollte und konnte bei der geringen Anzahl tüchtiger Lehrer in seinem Lande Nesthenus nicht entbehren ${ }^{2}$ ). Gnapheus

1) Der Herzog an den Elbinger Rat: „Es hat vnns vunser diener vnd lieber getreuer Jeronimus Pristinus Nestenus, nitverwannter vnd zugetaner der schulen jn vnser altenstadt Konigspergk, vnderthenigklichen zuerkenneu geben lassen, wie er sich für etlichen beygewichenen zeiten jn euren dienst gen Elbingen verpflicht mit demütigem bitten, jme solchs gnediglichen zugestatten vad sich derwegen zu euch zu begeben zuzulassen. Dyweill er denn für der zeit, vnd ehe sich gegen euch jn dienst verpflicht, inn unserem dienste verhaft vnd desselben bis auf diese stande nit gefreihet noch erlassen, haben wir seiner bit nit stadt geben wollen, sint auch noch zur zeit nit geneigt, jme darinnen zu wilfaren, sondern haben jme seinem dienste folge za geben erjnnert vnd derwegen darjnnen zu beharren bewogen. Ist derhalben hiemit an euch vuser gnediges begeren, nachdem gemelter Jeronymus sein thon bei euch vnbedechtlich fürgenommen, jr wollet jme solches zu keinem argen beimessen, besonders jn ferner jn seinem ersten dienst vnd verhaftnngen vngehindert beharren lassen. Dat. den 25. May $1536^{\prime \prime}$.

2) Wir haben euer schreiben, des datum steht Elbing, montags vor pfingsten des 36. jars, so jr auf rnnser ann euch ansgangene schrift wegen vnsers dieners Hieronimus Nesteni Phristinus an vns getan, empfangen vnd daraus euer dienstlichs bitten, das wir denselben Hieronimum dahin weisen wollten, seinem gelübde vnd verheißen, gegen euch bescheen, nachzukommen vnd davon nit zu weichen, sampt ferneren juhalt verstanden. Hierauf zweifeln wir nicht, jr habt euch aus jüngster vnser schrift zu erjnnern, welcher gestalt vns derselbe Hieronimus rerwandt. Dieweil wir denn mehrgemelten Hieronimum ebensowenig als jr entperen können, er vns auch zuror, vnd ehe er sich vieleicht gegen euch angezogener maßen dienstpflichtig gemacht, mit pflichten bis anf den heutigen tag verwandt rnd behaft rnd noch von vns (ob er wol vndertenig ersuchung bey uns gethan) solches seines dienstes verpflichtung nie keine erledigung erlangt, habt jr zu bedenken, wie seiner gegen euch bescheener zusage stadtzngeben vnd was gelegen sein will, eurer bit hirjn zu willfaren, insouderheit dyweil euch runverborgen, was die landesordnung jn einem artikel (do niemand jn behaftem dienst ohne vorgehenden beweislichen scheinlichen abschied abgefordert soll werden) diesfalls mitpringt, so jst an euch vuser gnediges begeren, jr wollet angezeigte vrsachen betrachten 
maßte sich nach einer anderen Kraft für sein Gymnasium umsehen. Er fand solche in Christoph Heyl, der, was ich zur Ergänzung der von Tschackert gebotenen Nachrichten bemerke, seit 1534 mit dem Herzoge Albrecht in Verbindung stand ${ }^{1}$ ).

Am 1. April 1538 sagte der Herzog dem Georg von Baysen za, auf seine Fürsprache den Adam Balinski, den er bereits eine Zeitlang in der Lehre hatte unterrichten lassen, wieder in die vorige Stelle za nebmen und zum Studium anzuhalten, fügt aber hinzu: ,Wiewohl itzund eine Schule für die Jugend zu Elbing stattlich gehalten werden soll und der Balinski dort besser mit der Lehre versorgt sein möchte". Er gab also dem Elbinger Gymnasium den Vorrang vor seiner Königsberger Schule. Er hatte Gnapheus schon im Sommer 1537 persönlich kennen gelernt, als er auf der Reise zur Krönungsfeier seines Schwagers Christian III. von Dänemark Elbing berihrt hatte. Damals hatte der Humanist einen seiner Schuler eine wohlausgearbeitete Begrubungsrede halten, andere Schüler wohlgefeilte Verse aufsagen lassen und damit besonderen Eindruck auf den Fürsten gemacht, der gerade nach solchem Hervortreten in der Oeffentlichkeit den Wert einer Schule und die Befähigung eines Lehrers bemaß. Dann hatte er in der Folgezeit gehört, wie der Adel seines Landes seine Söhne aufs Elbinger Gymnasium, an dem seit Sommer 1537 der aus Kolberg herangezogene Christoph Heyl griechisch lehrte, zu geben begann. Er selbst sandte dorthin im Mai 1539 den ältesten Sohn des Hauptmanns von Preußisch Eylau Kaspar von Rechenberg; Fabian. An unseren Gnapheas schrieb er unter dem 16. d. M.:

"Wir haben den Knaben schon eine Zeitlang und fast von Jugend auf an unserem Hofe erzogen und zur Schule wie andere vom Adel gehalten. Weil er aber nun zu verständigen Jahren gekommen, sein kranker Vater ihn uns befohlen, wir ihn auch gern als unseren eigenen

vnd vielgemeltem Hieronimo, ob er vielleicht dis stacks halben etwas vnbedechtlich vorgenommen, zu keiner leichtfertigkeit zumessen, sondern jn dasjenige, darza er sich erstmals alhie gegen vns vorpflicht vnd noch darjnnen behaft jst, abwarten lassen ... Dat. 6. Juni 1536.

1) Als der Herzog im Oktober 1534 zur Tagfahrt nach Driesen reiste, gab er Heyl anheim, zu ihm zu kommen, ${ }_{n}$ nachdem ... Joh. Poliander euch unseres Gemüts Meinung zugeschrieben, auch eure Antwort derhalben bekommen und abermal darauf eine Schrift an euch geschickt, aber nachmals eure Wiederantwort darauf nicht vernommen, woraus zunormutten, das euch vileicht solch letzt schreiben nicht behendiget". 
Sohn in der Zacht und Ehrbarkeit gefördert sehen wollen, haben wir ihn, dieweil ihr obnedies auch andere vom Adel bei euch habt, unter eure Zucht zu ton für geraten angesehen. Ihr wollet ihn in eure Disziplin nehmen und ihn gleich anderen Söhnen unserer Untertanen vom Adel mit Lager, Essen und Trinken unterhalten und an eurem FleiB, damit er und andere unsere Untertanen vom Adel, so bei euch, za gaten Tugenden, Zucht nnd Lehre auferzogen werden, nichts erwinden lassen."

Bald schickte der Herzog Gnapheus weitere Schüler za, so einen Schwerin, Balthasar Kannacher, Georg von Polentz, im Juni 1540 Georg Sachitzki, im Juli darauf Hans Gablentz, den Sohn des verstorbenen Gilgenburger Hauptmannes. Als er Sachitzki von Königsberg hinubersandte, schrieb er ${ }$ an den Schulmeister zu Elbing“"

nWir haben ihm zehn Mark, die er euch zar Kost geben soll, hier reichen lassen, die ihr also von ihm zu empfahen. Wir verlegen ihn zum Studio und hoffen, ihr werdet ihn wie andere anterweisen und euren Fleiß anwenden."

Auf des Herzogs Empfehlung kamen auch polnische Magnatensöhne zu dem mächtig aufblühenden Gymnasium, das nach einer Nachricht damals über 600 Schüler gezählt haben soll, unter ihnen Nikolaus Firlej ${ }^{1}$ ), der spätere Rawer Kastellan und Lubliner Wojewode. Balthasar Kannacher ließ sich zu einem hässlichen Ausbruch jugendlicher Auflehnung and Unbotmäßigkeit hinreisen, als Gnapheus ihm einmal zur Bestrafung mit dem Buche, das er gerade in der Hand hatte, gegen den Kopf geschlagen hatte. Der Herzog hörte davon, und gab dem Schuller einen strengen Verweis, tadelte aber auch Gnapheus, daß er nicht sofort Anzeige bei ihm erstattet habe. Er wünsche strenge Zacht and Ordnung, unbedingten Gehorsam; unbotmäBige Schüler werde er hinfort heimrufen and ihnen alle Unterstititung entziehen. Zagleich versichert er dem Rektor seiner Huld; es werde ihm eine Freude sein, wenn er eiumal ihn fördern und unterstützen könnte ${ }^{2}$ ).

Schon 1537 hatte Christoph Heyl, der Lehrer unter Gnapheus, dem Herzoge ein Büchlein zugeschrieben ${ }^{3}$ ), auch

1) L. Neubauer, Aus der Geschichte des Elbinger Gymnasiums S. 7, wo eines Schreibens Firlejs vom 26. April 1585 aus Lewartow gedacht wird.

2) Das Schreiben bei Wotschke, Herzog Albrechts Briefe an Laski. Altpreußische Monatsschrift XLV S. $473 \mathrm{f}$.

3) In Beantwortung eines Schreibens Heyls aus Kolberg vom 22. Febr. 1537 lässt der Herzog am 27. März ihm erwidern: „Das büchlein, so ir vas zuzuschreiben bedacht, sofern wir des zuvor ein exemplar bekommen vnd was materia vnd was es wäre vnd vns solches 
Anfang 1540 an den Herrscher sich gewandt wegen einer neuen Zueignung wohl einer astrologischen Deutung der Zukunft durch den Berliner Mathematiker Carion ${ }^{1}$ ), die freilich gerade keine günstige Aufnahme beim Herzog und seinem Hofe gefunden hat ${ }^{2}$ ). Nun wollte auch Gnapheas nicht zurïckstehen und dem Herzoge, der ihm so manchen Schüler zugewiesen, seine Dankbarkeit bezeugen. Am Weihnachtstage 1539 (er schreibt 40 , da er das neue Jahr mit dem Weihnachtsfeste anheben läßt) widmete er ihm seine Schulkomödie Morosophus, in der er den großsprecherischen Scheingelehrten verspottet, und sandte ihm die Handschrift zu. Am 6. Februar dankt der Herzog dem Humanisten in den anerkennendsten Worten. „Tuorum studiorum odorem universa redolet Borussia." Er ersucht ihn, die Komödie drucken zu lassen; für alle Kosten werde er aufkommen, und seine Schüler, die er schon hier und da auf Akademien hätte, würden gewiß zur Gewinnung eines

nach vbersehung gefiele, wollten wir alsdan, sofern es euch geliebt, in euren gefallen gestellt haben, dasselbe an vns ausgehen zu lassen". Als dann schon aus Elbing Heyl am 2. Juni geantwortet hatte, schrieb der Herzog den 26. Juni zurück: „Wir vermerken, daß jr ein büchleyn jn druck vnd, wenn es vns nicht zuwider were, an rns zu schreiben bedacht. Es ist rns solches, sonern es mit guthem reifen rath, geptirender fürsichtigkeit vnd beständigem grunde, wie wir denn nicht zweifeln, daß es euch mehr zu ehren dan zur vercleinerung gedrackt werde, nicht entgegen".

1) Den 17. April 1537 spricht der Herzog der Frau Margarete Carion sein Beileid zum Tode ihres Mannes aus. Kurz vor Weihnachten 1536 hatte Carion ihn um ein Empfehlungsschreiben an den Köuig von Dänemark gebeten. Da Carions Bote aber den Pfalzgrafen Ottheinrich nach Polen begleitet hatte, hatte der Herzog erst am 21. März den Brief von seiner Hand empfangen, an demselben Tage aber noch die gewünschte Empfehlung geschrieben.

$\left.{ }^{2}\right)$ Der Herzog unter dem 16. Febr. 1540 an Heyl: "Achtbar vnd hochgelehrter, lieber besonder! Wir haben vorschiner zeit ein schreiben von euch empfangen vnd dasselbe itznnd in ausreumung vasers gemachs zuhanden gekrigt, darjunen ir vns anzeigt, wie ir vns zo ehrenn vor dieser zeit ein buchlein Carionis zu Stettin habt dracken lassen vnd dafür fünff gulden ausgelegt mit feruerm juhalt. Hierauf fugen wir euch gnediger meynung wissendt, das wir vns aus dem aberschickten druck nicht wohl verrichten können vnd derwegen gern das original Carionis haben wollten." Unter dem 23. Sept. 1541: "Was anlangt Carionis buchlein, wissen wir noch bis auf diesen Tag nicht, wo das original hinkommen, möchten auch dasselbige nochmals gern bey vnseren händen haben. Denn der drack, so darnach gemacht, gefällt vns nicht, ist auch dem original jn allen stücken nicht gleichförmig." 
Drackers gern behilflich sein. Bei dieser Gnade des Herzogs gegen den Schulmann war es fast selbstverständlich, daß der Furst, der patronus omnium evangelicorum, wie ihn Laski einmal nennt, ihn freudig aufuahm und ihm ein Amt gewährte, als die Verfolgung durch den Bischof Dantiskus ihn Anfang Juli 1641 Elbing zu verlassen zwang. Auch Heyl stellte sich damals dem Herzoge zur Verfügung, falls er zur Beförderung der Studien eine höhere Schule gründen wollte, erhielt aber am 23. September eine ausweichende Antwort ${ }^{1}$ ); wurde aufs uubestimmte vertröstet auch ein Jahr später, als er von neuem an den Herzog herangetreten war ${ }^{2}$ ). Gnapheus Leben, Stellung und Wirken in Königsberg, auch seine Bekämpfung und schließliche Exkommunikation 1547 hat Tschackert unter Heranziehung des gesamten urkundlichen Materials gezeichnet. ${ }^{3}$ ) Ich habe hier nichts neues

1) Aus Lyck der Herzog unter dem 23. Sept. 1541: „Wir haben euer schreyben, ausgangen zu Königspergk den 6. Septembris, alhie in der wiltnuss bekommen. Wie jr nun auzeiget, das jr euch gerne, daß zu Königspergk die studia weitter möchten gefurdert werden, geprauchen lasset mit angehefter vndertheniger bit, euch, was wir hierjnuen zu thun gesinnet, zu verstendigen, wollen wir euch nicht pergen, dass wir noch disfals nichts entlichs mit vnsern landen vnd leuten beschlossen."

2) Der Herzog unter dem 30. Okt. 1542: „Wir haben ener schreiben, zum Elbinge dinstags nach Bartholomäi ausgangen, bekommen. Soviel nun erstlichen die danksagung vnserer guedigen verehrung betrifft, het es derselben gar nicht bedurfft, dan wir euch gnedigen willen zu beweisen nicht ungeneigt. Ferner euer gutwillig erbietten, vnd wie jr euch in vnserem dienst brauchen zu lassen gemeint, anlangend, darauf wollen wir each gnediger meinung nicht verhalten. Seintemal ruser vorhabend werk in aufrichtung eines stadtlichen particulars sein volkommenheit noch nicht erlangt, so wil vns noch zur zeit etwas meres gegen euch vber vnseren vorigen abschiedt vornehmen zu lassen unbequem seiu. Wan aber das particular volkomlichen aufgerichtet, alsdan wollen wir vns gegen euch auf weiter ansuchen vnserer gelegenheit nach aller gnedigen gebur zu erzeigen wissen." Auf ein erneutes Gesuch schreibt ihm der Herzog am 21. Aug. 1544: „In vnserem collegio sind itziger zeit alle lectiones dermaßen bestellt, das keine vakantz ist." Den 9. Juli 1555 empfiehlt ihn der Herzog dafür der Stadt Danzig als Arzt.

3) Uebersehen hat Tschackert hier nur ein Schreihen des Herzogs an den Rat zu Danzig vom 23. Juni 1547: „Es ist an vns gelangt, das Wilhelmus Gnapheus ... einen druck zur defension bey euch ausgehen zu lassen bedacht... Versehen vus, jr werdet jme ein solches zu uerhuttung ferneres beschwerlichen vnraths keineswegs vergönnen." 
ermitteln können. Als später dem Herzoge, der den Humanisten seinen Gegnern preisgegeben hatte, die Angen aufgingen, suchte er das Unrecht gut za machen. Er erlaubte ihm, 1554 nach Königsberg zu kommen und sich za rechtfertigen, schrieb daun auch, was Tschackert entgangen ist, an Staphylus, den einstigen Aktor im Prozesse wider ihn, sandte ihm die Prozeßakten ${ }^{1}$ ) und die Verteidigungsschrift des Gnapheus mit der Aufforderung sich eingehend zu äußern. Am 22. Oktober entschuldigte sich der ehemalige Professor der Albertina, der inzwiscben den Weg zurück nach Rom gefunden hatte, mit Mangel an Zeit, später werde er der Aufforderung entsprechen. Er hat es doch nicht getan. Darauf hob endlich 1561 der Herzog die Exkommunikation des Humanisten auf, gewährte auch seinem Sohne eine Unterstiutzung za seinen Studien. Am 29. Juli d. J. sandte ihm Gnapheus seinen Dank.

\section{Beilagen.}

Seit 1536 verhandelte der Herzog Albrecht mit dem Rate Elbings über das Dorf Reichenbach, in dem Paul Speratus die preaßische Kirchenordnung von 1525 noch nicht zur Geltung hatte bringen können, und deshalb Beschwerde bei ihm erhoben. Auf das herzogliche Schreiben vom 28. April entgegnete der Rat, daß die Jurisdiktion des Kirchspiels Reichenbach ${ }^{2}$ ) dem Kulmer Bischofe zustehe, worauf der Herzog am 7. Mai entgegnete, daß Reichenbach in seinem Herzogtume liege und kein fremdes Recht in ibm gelte. Er wiunsche, daß anch dort die für das ganze Herzogtum aufgerichtete Ordnung eingeführt werde. Die Stadt

Ein mir nicht näher bekannter Hans Konrad hatte sich im April 1547 für Gnapheus beim Herzoge verwandt und beklagt, eaß er in Ungnade gefallen sei. Am 23. antwortet ihm der Herzog: „Wenn sich Gnaphens dem Abschiede gemäß erzeigt, soll er als ein Christ, der im Glauben und Lehre mit uns einig, bei uns in allen Guaden sein."

1) Wotschke, Aus Herzog Albrechts Briefwechsel mit Schlesien. Korrespondenzblatt 1908 S. $21 \mathrm{ff}$, dort auch des Staphylus Antwort.

2) Unter dem 6. Sept. 1539 ersucht der Herzog Zehmen, das Dorf Altendorf, das sich vom Kirchspiel Altstadt gelöst habe, wieder zur alten Parochie za halten. Ohne Altendorf könne Altstadt keinen Pfarrer ernähren. An demselben Tage bittet er Werden ain Auskunft, ob die Kirche in Alt Christburg abgebrochen werden könne. Das baufällige Gebäude sei zur Instandsetzung der auch baufälligen Kirche in Altstadt erbeten. 
solle den Anordnungen des Bischofs Speratus Folge leisten, sonst mußte er einschreiten. Jobannisburg, den 24. Dez. 1537 schreibt er an Hans von Werden:

"Vns ist zu wissen vonnöten, wie vnd welcher gestalt der stadt Elbing das dorff Reichenbach verschrieben. Darumb, weil solchs zweifels on in dem handtuestenbuch za Preuschenmargk verzeichnet, wollet vns des ein abschrift widerfaren lassen, oder aber, woe die nicht vorhanden, bey denen von Elbing, oder sonst wo die zu bekommen ist, vns eine glanbwidrige abschrift zuwege bringen."

Memel, den 15. April erwidert der Herzog anf Werdens Antwort rom 14. April :

„Wir haben verstanden, wie die Elbinger zam dorff Reychenbach kommen. Und weil wir jn allen vnseren emptern handtrhestenbücher aufrichten haben lassen, dariu man alte vnd newe handvhesten schreyben möge, so begeren wir, jr wollet die handvhesten vber das dorff Reychenbach von denen zu Elbing in vnserem namen fordern vnd jn das handtrhestenbuch des preußischmerkischen ampts schreyben lassen, auch vns eine glaubwidrige copey derselbigen zuschicken."

Pr. Holland, den 28. Juli 1538 schreibt schließlich der Herzog nach Elbing, noch sei in Reichenbach kein Pfarrer, der den Armen mit der christlichen Lehre vermöge der preußischen Kirchenordnung vorstehe und sie mit dem $\mathbf{h}$. Abendmahl versorge. Speratus werde jetzt einen Pastor fur dieses Dorf ordinieren und senden, Elbing möge seine Untertanen zu allem Gehorsam wider ihn anhalten.

Den 14. März 1543 beschwert sich der Herzog, daß Elbing dem Pfarrer $^{1}$ ) Ambrosius Feierabend in Weinsdorf

1) Für den Tapianer Pastor Paul Grunwald, der 1526 aus Danzig vom Leslauer Bischof vertrieben war, wendet sich der Herzog am 15. Juni 1537 an Achatius von Zehmen mit der Bitte, ihm von Danzig einen Geleitsbrief za verschaffen, $\mathrm{daB}$ er sein $\mathrm{Hab}$ und Gut von dort holen könue, schreibt deshalb auch am 3. Juni des folgenden Jahres an Hans von Werden. Direkt an den Danziger Rat schreibt der Herzog am 25. Juli 1545 für den gleichfalls 1526 verjagten Prediger an der Barbarakirche Jakob Möller. Unter dem 24. Jnni 1043 bekundet der Herzog Zehmen seine Freude, daß er neben dem Kulmer Bischof Tiedemann Giese als Kommisar in Glaubenssachen nach Danzig verordnet sei. „Wären gern verständigt, welcher Gestalt der großmechtige wnd wohlgeborene vnser besonder lieber freundt vnd bruder her Stanislaus Odrowasch, graf zu Jarasslaw, ron dem hern ertzbischof des glaubens halben angetastet." Memel, den 21. April 1538 meldet der Herzog Hans von Werden, daß er auf sein Gesuch die Söhne seiner Schwester and seinen Schwager, die nach Wittenberg gehen wollten, um christliche Künste und gute Tugend zu lernen, dem Kurfürsten von Sachsen empfohlen habe, ferner am folgenden 11. Mai, daß 
um des Evangeliums willen seine Habe vorenthalten, bei seinem letzten Aufenthalte in der Stadt ihn nicht zu hausen geboten hätte. Er fordert, seinem Untertan Gerechtigkeit widerfahren zu lassen.

\section{Wilhelm Gnapheus an Herzog Albrecht.}

Dici non potest, serenissime princeps, clementissime domine, quanto me gaudio tuae literae exhilararint, quibus et conciliationis

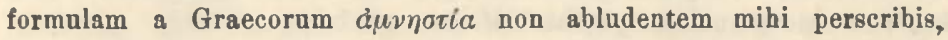
qua me vestrae ecclesiae restitui posse pie ostendis et clementiae quoque tuae significatione in eo mihi liquido declaras, quod filium meum biennium adhuc totum tuis sumptibus in literis apud Germanos alere non detrectes. Ut itaque priori epistolae tuae parti respondeam, T. Cels. latere nolim, princeps optime, quod cum nihil aeque in votis habeam, quam ut cum ecclesia sancta dei, quae apud vos est, etsi illa, quod ego quidem sciam, nunquam a me laesa sit, in gratiam redeam, nihil prius mihi faciendum duco, quam at propositas mihi a T. Cels. conciliationis leges accipiam, veneror et amplector. Quis enim non praestabilius tecum indicet, princeps illustrissime, at vel sancta oblivione deleatar vel unius dei indicio committatur, si quid ab alterutra parte in alteram commissum sit, quam ut nova contro. versia niminm altercando iis moveatur etiam, qui rebus humanis exempti sunt, cum iuxta proverbium cum mortais non luctentur nisi larvae. Ea propter $T$. ego Cel. enixe precor, ut, si absente me haec reconciliatio sive restitutio mei cum vestra ecclesia commode procedere possit, tua clementia eam sic institui per ecclesiarum ministros istic iubeat, ut non nisi honorificum mihi sit clementiae tuae indicio hac in re acquierisse me. Neque enim ab re fuerit meo quidem iudicio, princeps optime, si universum huius controversiae odium in anta. gonistam meum Staphilum, hominem impurum, ne quid gravius in illum dicam, reiciatur, cum ad meam apologiam per T. Clementiam illi ante sexennium missam quicque respondere in hunc usque diem tanıquam male sibi conscius contumacissime recusarit. Eam autem mei cum ecclesia vestra restitutionem, ubi ex Cels. T. praescripto $\mathrm{f}_{\mathrm{acta}}$ fuerit, literis tnis testimonialibus latine conscriptis tali formula, quae mihi honorifica sit, palam omnibus significari velim, ut ne vel mihi vel liberis meis in posterum fraudi sit vel probro etiam obvertatur, quod tam indigna excommunicationis censura ex machinatione

er auf seine erneute Bitte auch Peter Behem, den nach Wittenberg gehenden Sohn des Danziger Bürgermeisters in das Empfehlungsschreiben aufgenommen habe. Vierzehn Tage später: ${ }_{n}$ Vns haben die zwene ehrliche christliche bidermenner ehr Martin Luther vnd Philippus Melanton diese jnliegende zeitung zugeschrieben". Einen Druck, den ihm Melanchthon zugleich gesandt, werde er erst lesen, dann auch Werden schicken. 
satanae haud dubie antea istinc eiectus fuerim. Tum id quoque iisdem Cels. T. literis omnibus cupiam innotescere T. Cels. iusta de cansa mihi in priorem nominis mei existimationem restituto eam facultatem perbenigne concessisse, ut libere ac tuto in principatus tai terras redeam et pro meo arbitratu istic agam, maneam, verser et, si videatur, domicilium etiam statuam, quemadmodum omnibus aliis Cels. T. subditis hoc liberum existit.

Quod vero alteram epistolae tuae partem attinet, magno certe beneficii loco accipio, princeps optime, quod filium meum Albertum, alumnum tuum, in aliqua academia per Germaniam bene constituta tuis sumptibus biennio adhuc toto alere liberaliter adeo et perbenigne offeras, ut hac ratione rerum mearum damnum (quod scio, quod mibi ob hoc exsilium fuerit eximium et irreparabile) aliqua saltem in parte mihi sarciatar. Cum itaque eam mihi liberalitatem offeras et filius ille meus ea nunc sit aetate eumque progressum in literis fecerit, ut omnes iudicent tempestivnm esse, quo alibi quoque stadia sua provehere pergat, T. ego Cels. enixe obsecro, ut quam mihi munificentiam per literas benigne adeo obtulisti, nunc re ipsa praestes et filium illum meum annuo commeatu a T. Cels. benignitate donatum pariterque literis tuis commendatitiis instructum hoc praesenti autumno ad me redire iubeas, ut primo qnoqne tempore a me ille Marburgum mittatur, ut ubi illic aliquod operae pretium in literis fecerit, elapso anno inde alio quopiam ex T. Cels. judicio se recipiat. Ea enim spe hic tuus alumnus mihi nunc esse videtur, ut de eo metuendum non sit, ne oleum simul et opera, quod dicitur, perdantur, nisi mea me fallat philantia.

Constitueram quidem, serenissime princeps, pro magno animi mei in te studio Cel. T. invisere, ut tecam liceret super variis rebus commentari et cum amicis studiorum nostrorum memoriam refricare, sed Rhanusia ipsa eam mihi foelicitatem, ut opinor, invidit. Cum enim ineunte aestate comitum nostrorum, in quorum ego nunc sum fide, negotia hic me diutius retinuissent, quam vel ipse vellem vel constitutae profectioni in Borussiam conveniebat, evenit, ut procedente nunc adnlta aestate, si istic progrediar, ante hiemis adventum huc redire nequeam, quae causa est, cur per literas nunc utcumque agere cogar, quod alioqui coram agere, si licuisset, maluissem. Quam necessitatem meam ut Cels. T. non minus aetati meae iam ingravescenti quam temporis angustiis ignoscat, oro quaesoque. Quod superest antem, princeps optime, venerandam tuam senectutem, quam reverenter colo, cum iuniore principe et filia omnibusque tuis deo opt. max. commendo. Nordae Frisiorum 29. Julii a. 1561. Ill. Cels. T. deditissimus cliens Gulielmus Gnapheus. 


\section{Mitteilungen.}

\section{Nenerscheinungen.}

\section{Zum Augustanajubiläum.}

„Die Glaubensartikel der Augsburger Konfession, erläntert von Jul. Köstlin ", zuerst 1891 unter den Schriften für das deutsche Volk erschienen, ist vom Verein f. Ref.Gesch. zum Jubiläım nochmals ansgegeben worden. Das Schriftchen legt von sicherer wissenschaftlicher Grundlage aus den Inhalt der Bekenntnisschrift klar und schlicht für einen möglichst weiten Kreis von Lesern aus und bietet so die beste Einführung in Inhalt und Verständnis der Konfession. $101 \mathrm{~S}$. kl. 80. Leipzig, M. Heinsius Nachf., 1930. M. 0,60 .

W. Vollrath, „Das A ags urger Bekenntnis und seine Bedeutung für die Gegenwart", würdigt die Konfession als Bekenntnis, als Verantwortung und Rechenschaft, woraus sich ihre Bedeutıng für die Gegenwart ohne weiteres ergibt. Zuerst werden die Umstände gezeichnet, unter denen die K. entstand und überreicht wurde, dann Luthers Einwirkung besprochen, endlich die K. selbst nach Form und Inhalt gewürdigt. Zum Schluß ein Blick auf die Gegenwart. Leipzig, Deichert (W. Scholl). $78 \mathrm{~S}$. $8^{0}$. M. 2.50. (Jubiläumsgabe der allg. Ev.-Luth. Konferenz)

Eine Vorstufe zur A ugs urgisch $\in$ n $K$ onfession behandelt das vom Landeskirchenrat der Ev.-Luth. Kirche in B a yern r. d. Rh. herausgegebene Werk „Die fränkischen Bekenntnisse". Es zerfällt in Untersuchungen von Wilh. Ferd. Schmidt und Texte von $K$. $S \mathrm{ch}$ ornbaum, das ganze angeregt und gefördert von H. $\nabla . \quad S \mathrm{chubert}$. I. Die Untersuchungen (S. 3-152) sind dogmengeschichtlicher Art; sich auf das amtliche Material beschränkend behandeln sie 1. den Ansbacher Biblizismus und die Gntachten der kleineren fränkischen Stände $1524 / 25$; 2. Die Osiandrische Theologie des Wortes in Nürnberg 1524/25; 3. Die Lehre der fränkischen Theologen bis zur Augsb. Konfession. II. Die Texte (S. 153-655) geben Abdrücke, vollständig oder im Auszug, von 26 der zahlreichen Bedenken und Gutachten aus Franken (ein Verzeichnis führt 85 fränkische Bekenntnisse aus den 